\title{
Inactivation of CGKI in the CNS causes adaptation defects in the circadian clock in mice
}

\author{
Sonja Langmesser*1, Susanne Feil ${ }^{2}$, Franz Hofmann² ${ }^{2}$, Robert Feil ${ }^{2}$ and \\ Urs Albrecht ${ }^{1}$
}

\author{
Address: ${ }^{1}$ Department of Medicine, Institute of Biochemistry, University of Fribourg, Switzerland and ${ }^{2}$ Institut für Pharmakologie und Toxikologie, \\ Technische Universität München, Germany \\ Email: Sonja Langmesser* - sonja.langmesser@unifr.ch \\ * Corresponding author
}

from 2nd International Conference of cGMP Generators, Effectors and Therapeutic Implications

Potsdam, Germany, 10-12 June, 2005

Published: 16 June 2005

BMC Pharmacology 2005, 5(Suppl I):P3 I doi:I0.||86/|47|-22I0-5-SI-P3 |

The cGK family of cGMP-dependent protein kinases, also known as PKG, has been described to be involved in the regulation of a broad variety of physiological processes. More recently, cGKI and II have been implicated in the resetting of the master circadian clock located in the suprachiasmatic nuclei (SCN). Notably, cgkII knockout mice show reduced phase delays and reduced Per2 induction in the early subjective night. These results are in contrast to studies using pharmacological cGK inhibitors. Since in mice, both cGKs are expressed in the SCN, we set out to investigate the wheel running behavior of $c g k I$ knockout mice to study the possible involvement of cGKI in resetting the circadian clock in mice.

Conditional $c g k I$ knockout mice were generated by introducing a conditional $c g k I$ allele carrying two loxP sites together with the Cre recombinase under the control of the nestin promoter which results in an excised inactive allele in the central nervous system. Absence of cGKI in the hypothalamus was confirmed by immunohistochemistry. To assess the functionality of the circadian clock in these mutant mice, their wheel running behavior in different lighting schedules as well as their response to phaseshifting stimuli were tested.

Conditional $c g k I$ knockout mice showed normal entrainment to LD cycles and an internal period length comparable to that of control animals under free-running conditions. No change could be observed in behavioral phase shifts, clock gene induction in the SCN and phosphorylation of CREB at Ser32 in the retina after administration of light pulses at CT10, 14, and 22. Nonphotic cues also had the same effect on both genotypes. However, mutants displayed difficulties in entraining to new lighting schedules. Mutants had a higher onset error than control animals, and a markedly higher percentage of daytime activity was measured. Additionally, conditional cgkI knockout mice had a longer transition time after light pulses at CT22. Using a lighting schedule mimicking nonchronic jet lag, mutants adapted to the changes as fast as control animals, but a second, free-running rhythm could be observed. Under chronic jet lag conditions, the activity was more fragmented in mutants than in the controls, and total activity was reduced after release into DD. Thus, the mutants appeared to be more disturbed by the rapid changes in the lighting schedule.

In conclusion, our analysis of conditional $c g k I$ knockout mice indicates that CGKI in the brain does not play a crucial role in resetting of the circadian clock. It seems more likely that cGKI is required for an efficient adaptation to changes in the lighting schedule as well as for fine-tuning of the clock oscillator. 